\title{
Physical-mechanical performance of the concrete produced with fiber addition jute plant in the partial replacement of Portland cement in $0.5 \%$ and $1.0 \%$
}

\author{
Bianka Caroline Cunha Firmino ${ }^{1}$, Murilo Ferreira dos Santos $^{2}$, Darlei dos anjos Lavor ${ }^{3}$ \\ David Barbosa de Alencar ${ }^{4}$, Larissa de Sá Sousa ${ }^{5}$, Edson Andrade Ferreira ${ }^{6}$ \\ $1,3,5,6$ \\ Centro universitário do Norte (UNINORTE). Manaus-AM. \\ Instituto de Ensino Superior Blauro Cardoso de Mattos (FASERRA). Manaus-AM. \\ ${ }^{4}$ Instituto de Tecnologia Galileo da Amazônia (ITEGAM). Manaus - AM, Brasil.
}

Email: biankakarolinne@hotmail.com, mullsantos@gmail.com, darleilavor27@gmail.com, david002870@hotmail.com, edson.ferreira@uninorte.com.br, larissasousa490@gmail.com

Received: January $13^{\text {th }}, 2018$

Accepted: February $14^{\text {th }}, 2018$

Published: March $31^{\text {th }}, 2018$

Copyright (2) 2016 by authors and Institute of Technology Galileo of Amazon (ITEGAM) This work is licensed under the Creative Commons Attribution International License (CC BY 4.0).

http://creativecommons.org/licenses/by/4.0/ (c) (1) (3) Opean Acees:

\begin{abstract}
This work presents a feasibility study of the use of Jute fibers (Corchurus capsularis) as reinforcement in the partial replacement of Portland cement in conventional concrete with fck of 25 $\mathrm{MPa}$. For this, during the study stages, numerous laboratory tests were carried out with the purpose of characterizing the materials used in this research, both for the small aggregates and for the Portland cement and the proposed fibers. The objective after the characterization of the materials was to investigate the physical and mechanical behavior of the concrete specimens molded for the analysis of rupture at 7 and 28 days of curing and water absorption, divided into three different groups, a molding being the pilot with $0.0 \%$ addition of fibers denominated as reference and another two with different percentages extracted from the consumption of total cement used in concrete test specimens under analysis, being group 2 with $0.5 \%$ and group 3 with $1,0 \%$ fiber.
\end{abstract}

Keywords: Concrete, Jute Fiber, Potland Cement.

Desempenho físico-mecânico do concreto produzido com adição de fibra vegetal de juta na substituição parcial do cimento Portland

$$
\text { em } 0,5 \% \text { e } 1,0 \%
$$

\section{RESUMO}

Este trabalho apresenta um estudo da viabilidade de utilização das fibras de Juta (Corchurus capsularis) como reforço na substituição parcial do cimento Portland em concreto convencional com fck de $25 \mathrm{MPa}$. Para isso, durante as etapas do estudo, foram realizados inúmeros ensaios laboratoriais com a finalidade de caracterizar os materiais empregados nesta pesquisa, tanto para os agregados miúdo e graúdo quanto para o cimento Portland e as fibras propostas. O objetivo após a caracterização dos materiais foi investigar o comportamento físico e mecânico dos corpos de prova de concreto moldados para as análises de ruptura aos 7 e 28 dias de cura e de absorção de água, divididos em três grupos diferentes, uma moldagem sendo o piloto com $0,0 \%$ de adição das fibras denominado como referência e outros dois com diferentes porcentagens extraídas do consumo de cimento total utilizado nos corpos de prova de concreto em análise, sendo o grupo 2 com $0,5 \%$ e o grupo 3 com $1,0 \%$ de fibra.

Palavra-chave: Concreto, Fibra de Juta, Cimento Potland. 


\section{INTRODUÇÃO}

Adaptar materiais recicláveis na construção civil tornouse um pré-requisito sobre sustentabilidade e

economia. É imprescindível não notar em um canteiro de obra a coleta seletiva dos materiais descartados dos quais são classificados como Resíduos da Construção Civil (RCC). Pensando na escassez de matérias primas, inúmeras pesquisas foram desenvolvidas com os RCC racionando a utilização dos materiais naturais e adaptando os RCC, tais quais em concretos, solos e/ ou asfaltos. Com vista nessas possibilidades, surgiram materiais novos adaptados a partir desses resíduos. Um grande exemplo deles são os compósitos de matrizes cimentícias.

Um material compósito é a combinação de dois ou mais materiais que têm propriedades que os materiais componentes isoladamente não apresentam. Eles são, portanto, constituídos de duas fases: a matriz e o elemento de reforço, e são desenvolvidos para aperfeiçoar os pontos fortes de cada uma das fases [1].

Os compósitos à base de cimento reforçado com fibras na produção de argamassas surgem como uma nova tecnologia a fim de se obter um ganho de produtividade e redução de custos devido as fibras conferirem um bom comportamento mecânico ao compósito [2].

A adição de fibras nas matrizes pode melhorar as suas propriedades mecânicas, como a resistência à tração, à flexão e ao impacto. Além disso, altera seu comportamento após fissuração diminuindo os efeitos de uma ruptura brusca da matriz cimentícia [3].

As matrizes cimentícias são compostas de aglomerantes minerais, podendo conter agregados, que dão origem as pastas, argamassas ou concretos. As matrizes mais utilizadas são aquelas à base de cimento Portland e, em menor escala, a cal e o gesso [4].

Nos últimos anos a produção de artigos e pesquisa referente à compósitos utilizando fibras vegetais aumentou consideravelmente. Entretanto, compósitos reforçados com fibras vegetais ainda estão na dependência de alguns fatores importantes, relacionando a sua aplicação e desempenho. É importante considerar que as fibras vegetais têm composição química diferente e depende do tipo de planta, da dimensão da célula cristalina, do ângulo helicoidal que a celulose faz em relação ao eixo central, defeitos superficiais, estrutura da macrofibra vegetal, propriedades físicas e mecânicas das fibras e a interação que a fibra pode fazer com a matriz do compósito [5].

Uma pesquisa que observou a durabilidade de matrizes cimentícias com fibra de coco comparou fibras novas com fibras presentes em uma argamassa utilizada em uma parede construída 12 anos antes [4].

A produção de coco, em toneladas, no Brasil saltou de 1.300.000 no ano 2000 para quase 2.000.000 de toneladas em 2010. Esta grande e crescente quantidade de coco propicia a utilização de suas fibras para diversas finalidades [6]. Dentre elas, a incorporação (através de adição) em matrizes de cimento portland vem sendo bastante estudada, pois sua aplicação pode melhorar as propriedades mecânicas dos compósitos cimentícios [7].

As fibras naturais vegetais são uma opção como reforço de matrizes cimentícias, principalmente após a proibição dos produtos de cimento reforçados com fibras de amianto, devido à comprovação de riscos à saúde. As fibras naturais são um recurso renovável e estão disponíveis em quase todo o mundo, além do baixo custo, quando comparada a utilização de fibras sintéticas que exigem maior consumo de energia e, portanto, um maior custo de produção [8].

A construção civil tem apresentado mudanças que contribuem significativamente para a melhoria na qualidade da cadeia produtiva, por meio de exigências contidas nas normas técnicas, redução do desperdício em canteiros de obras, utilização de sistemas industrializados e a formação de um sistema nacional de certificação [9]

\section{MATERIAIS E MÉTODOS}

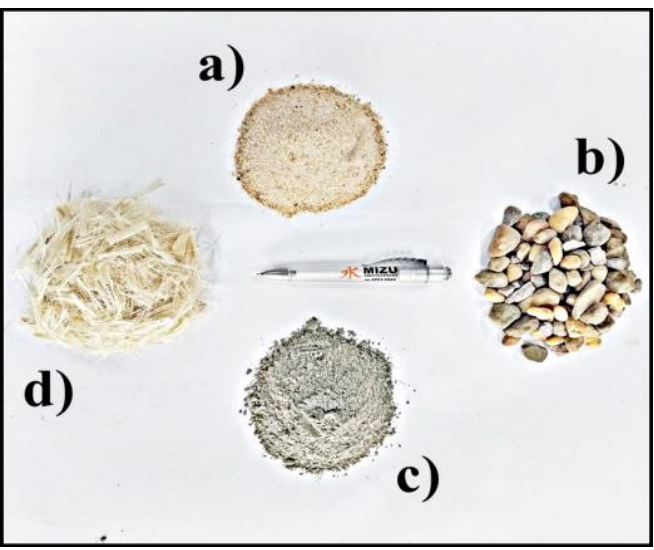

Figura 1: Materiais utilizados para a mistura do concreto estudado nesta pesquisa - a) agregado miúdo (areia fina); b) agregado graúdo (seixo rolado); c) Cimento CP IV-32; d) Fibras de Juta.

Fonte: Autores, (2018).

\subsection{PROCESSO DE SELEÇÃO E PREPARAÇÃO DA FIBRA}

A fibra utilizada para essa pesquisa foi doada por um morador do município de Manacapuru extraída da própria cidade onde é considerada uma das maiores beneficiadoras de sacos produzidos com este produto. Os materiais ainda em ramos foram secos em estufa a $70^{\circ} \mathrm{C}$ em um período controlado de 60 minutos, após o termino da secagem optou-se por uma seleção manual do material separando resíduos e deixando apenas a parte desejada como mostra a figura 1.

Todo o processo do corte ao desmanche foi feito de forma artesanal e cuidadosa para que cada fio se aproximasse da medida adotada de $2 \mathrm{~cm}$, ainda nesta etapa foram separados fio a fio e o resultado final foi um produto que se assemelhou as fibras já encontradas no mercado.

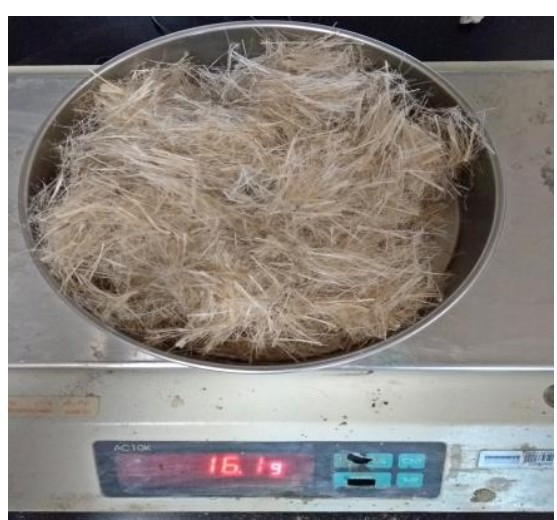

Figura 2: Fibra de Juta selecionada e cortada no tamanho de 20 mm preparada para a confecção do concreto.

Fonte: Autores, (2018). 


\section{II.2 CIMENTO UTILIZADO}

Nesta pesquisa o cimento utilizado foi o modelo CP-IV 32 Super Forte da marca MIZU. Optou-se por esse produto por ser um dos cimentos mais usados em pequenas construções e de fácil acesso na cidade de Manaus. Foram feitos vários testes para avaliar a viabilidade do material para esta pesquisa como: Determinação da massa específica segundo a NBR 16605 Cimento Portland e outros materiais em pó; Determinação da Finura; NBR 11579 - Cimento Portland - Determinação do índice de finura por meio da peneira $75 \mu \mathrm{m}\left(\mathrm{n}^{\circ} 200\right)$, de 2012; Determinação da pasta de consistência normal; NBR 16606 Determinação da pasta de consistência normal, de 2017 e Determinação dos tempos de pega; NBR 16607.

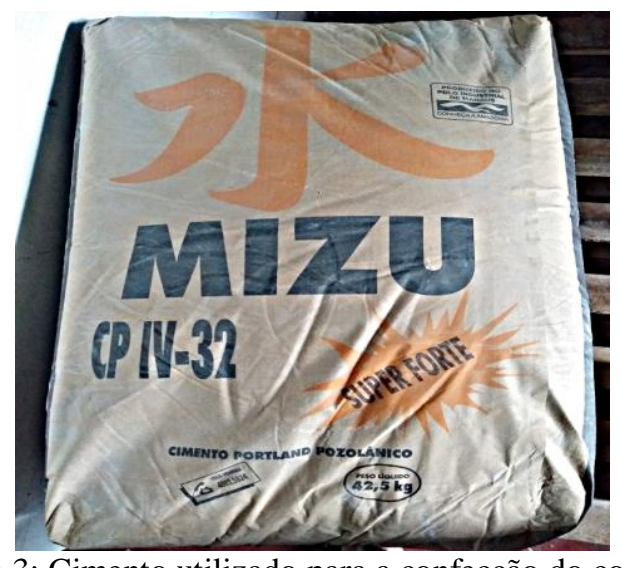

Figura 3: Cimento utilizado para a confecção do concreto estudado na pesquisa.

Fonte: Autores, (2018).

\section{II.3 CARACTERIZAÇÃO DOS AGREGADOS: MIÚDO E GRAUDO}

Seguindo as premissas técnicas encontradas na NBR 7211/2005 e 7211/2009 - Agregados para concreto - Especificação. O processo e caracterização dos agregados que compõe o concreto convencional para atender as especificações do método adotado para este estudo se deu utilizando 3 normas fundamentais listadas a baixo. $\mathrm{O}$ seixo e a areia utilizada nesta pesquisa foram comprados em uma loja de materiais de construção na zona centro Sul da cidade de Manaus.

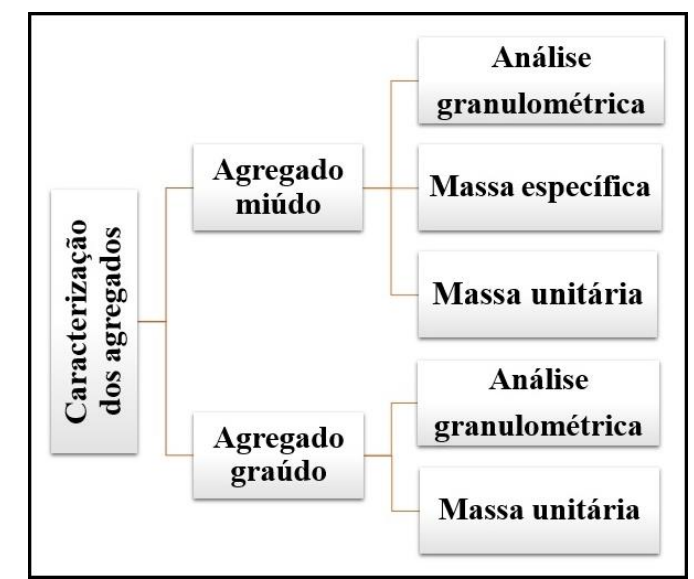

Figura 5: Fluxograma das atividades realizadas para caracterização dos agregados graúdo e miúdo.

Fonte: Autores, (2018).

\section{II.3.1 NBR NM 248:2003 - AGREGADOS - DETERMINAÇÃO DA COMPOSIÇÃO GRANULOMÉTRICA}

A análise granulométrica elaborada tanto para o seixo quanto para a areia foi feita seguindo todas as especificações necessárias em uma sequência de oito peneiras e o fundo, como manda a NBR 248:2003, em uma média retirada de três amostras.

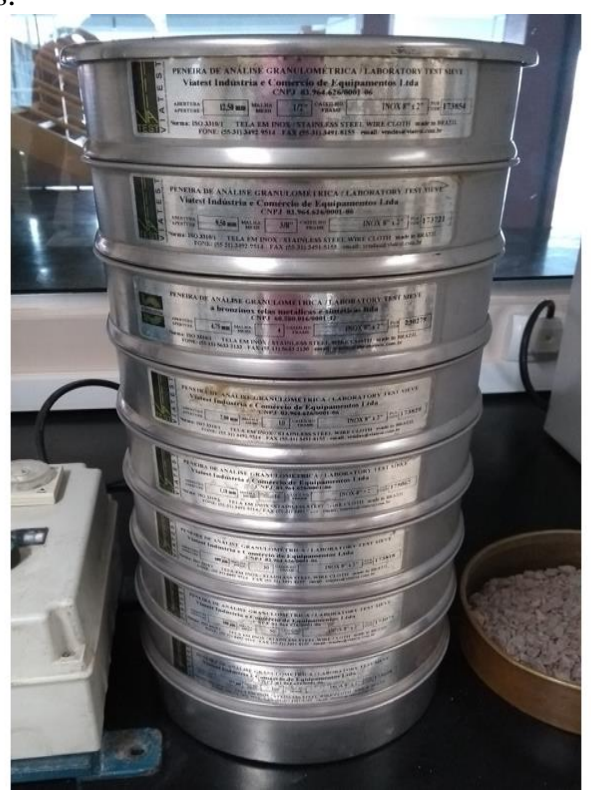

Figura 5: Peneiras utilizadas para o ensaio de caracterização dos agregados miúdo e graúdo. Fonte: Autores, (2018)

\section{II.3.2 NBR NM 45:2006 - AGREGADOS - DETERMINAÇÃO DA MASSA UNITÁRIA E DO VOLUME DE VAZIOS}

A análise da determinação da massa unitária para os agregados miúdo e graúdo seguiram as especificações necessárias abordadas na NBR NM 45:2006. O frasco utilizado em material de inox possui $372,4 \mathrm{~g}$ e um volume de $1553,5 \mathrm{~cm}^{3}$. Foram feitas três análises de cada material e em seguida extraído a médias de ambos de acordo com as exigências. A figura 6 mostra um dos momentos da execução do teste de massa unitária do seixo rolado.

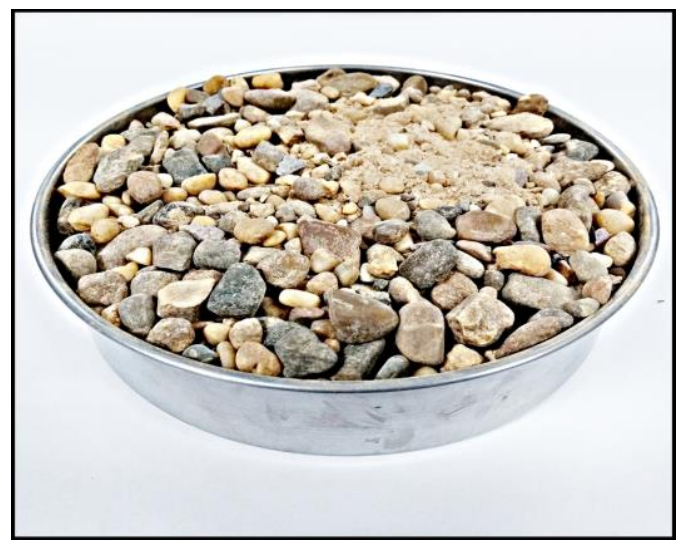

Figura 6: Recipiente utilizado para análise da massa unitária dos agregados miúdo e graúdo. Fonte: Autores, (2018). 


\section{II.4 NBR NM 52:2009 - AGREGADO MIÚDO - DETERMINAÇÃO DA MASSA ESPECÍFICA E MASSA ESPECÍFICA APARENTE}

Para a execução da determinação de massa especifica do agregado miúdo utilizou-se o Frasco Chapman seguindo as especificações da NBR NM 52:2009, perfazendo um total de 3 análises do material seco e por fim retirando a médias chegando no valor apresentado de $2330,0 \mathrm{~cm}^{3}$.

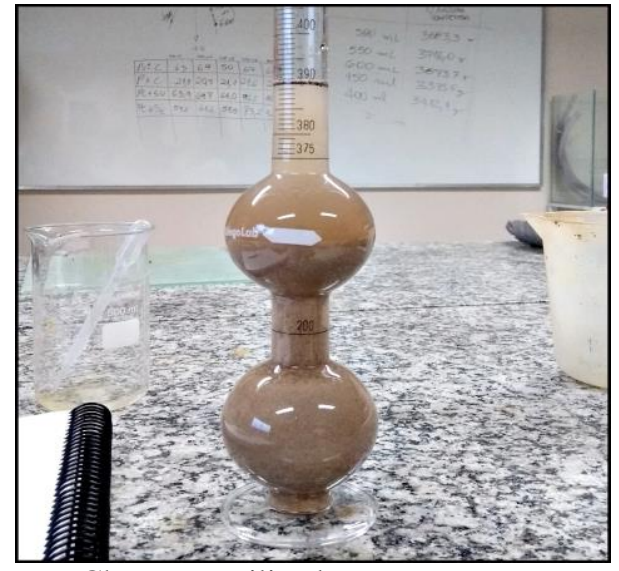

Figura 7: Frasco Chapman utilizado para executar o processo de determinação da massa específica do agregado miúdo.

Fonte: Autores, (2018).

\section{II.4 ÁGUA}

A água utilizada tanto para a moldagem dos corpos de prova quanto para a cura é proveniente do abastecimento público da cidade de Manaus, MANAUS AMBIENTAL S/A.

\section{II.5 MISTURA, MOLDAGEM, CURA E RUPTURAS DOS CORPOS DE PROVA}

O processo de mistura do concreto foi executado de forma mecânica em betoneira disponibilizada pelo Laboratório de Materiais de Construção do Centro Universitário do Norte seguido pela primeira etapa de moldagem dos corpos de prova, o desmolde dos mesmos foi feito 24 horas após a primeira etapa e colocado em cura úmida até os dias estipulados para a ruptura em 07 e 28 dias. Essas etapas seguiram as premissas técnicas estipuladas pela NBR 5738:2015 - Concreto - Procedimento para moldagem e cura de corpos de prova.

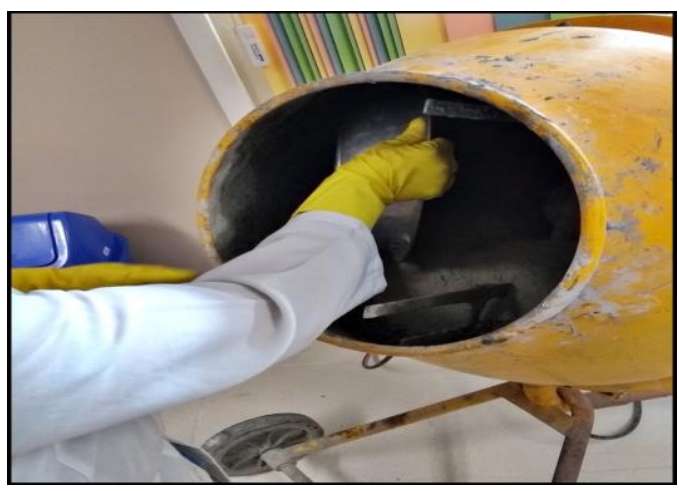

Figura 8: Betoneira utilizada para a mistura rápida do concreto convencional utilizado no desenvolvimento da pesquisa. Fonte: Autores, (2018).

\section{RESULTADOS E DISCUSSÕES}

Para que chegássemos aos resultados mais precisos nesta etapa a dosagem de cimento e dos agregados foram estabelecidas seguindo os padrões estipulados pela Associação Brasileira de Cimento Portland (ABCP). A Tabela 1 mostra os resultados obtidos através das análises e caracterização dos materiais usados para a mistura do concreto, todos os materiais foram devidamente selecionados em função do peso inclusive a água.

Tabela 1: Características e informações preliminares dos materiais utilizados para a dosagem do concreto

\begin{tabular}{|c|c|}
\hline \multicolumn{2}{|l|}{ CONCRETO } \\
\hline Fck (Mpa) & 25 \\
\hline \multicolumn{2}{|l|}{ CIMENTO } \\
\hline Resistência normal aos 28 dias (MPa) & 32 \\
\hline Massa específica $\left(\mathrm{Kg} / \mathrm{m}^{3}\right)$ & 3100 \\
\hline \multicolumn{2}{|l|}{ AGREGADO MIÚDO } \\
\hline Módulo de finura & 2,51 \\
\hline Massa específica Areia $\left(\mathrm{Kg} / \mathrm{m}^{3}\right)$ & 2630,0 \\
\hline Massa unitária Areia $\left(\mathrm{Kg} / \mathrm{m}^{3}\right)$ & 1513,0 \\
\hline \multicolumn{2}{|c|}{ AGREGADO GRAÚDO } \\
\hline Dimensão Máxima característica (mm) & 12,5 \\
\hline Massa específica Seixo $\left(\mathrm{Kg} / \mathrm{m}^{3}\right)$ & 1990,0 \\
\hline Massa unitária Seixo $\left(\mathrm{Kg} / \mathrm{m}^{3}\right)$ & 1825,0 \\
\hline
\end{tabular}

Fonte: Autores, (2018).

\section{III.1 CRONOGRAMA DAS ATIVIDADES DESENVOLVIDAS ENTRE OS PROCESSOS DE MISTURA AO ROMPIMENTO}

O cronograma foi cuidadosamente elaborado e seguido de acordo com as normas já citadas no item III.5 para que atingisse o máximo de excelência no resultado final das amostras, para a execução das misturas adotou-se propositalmente a diferença de um dia já que para cada dosagem dos materiais utilizados nos traços os pesos eram diferenciados, atribuindo a esta etapa a atenção redobrada na elaboração.

Tabela 2: Cronograma das etapas de mistura, moldagem, desmolde, cura e ruptura dos corpos de prova aos 07 e 28 dias

\begin{tabular}{|c|c|c|}
\hline \multicolumn{3}{|c|}{ Cronograma das atividades executadas } \\
\hline TRAÇOS & $\begin{array}{c}\text { DATA DE } \\
\text { MOLDAGEM }\end{array}$ & SUBMERÇÃO \\
\hline REFERÊNCIA & $02 / 07 / 2018$ & $03 / 07 / 2018$ \\
\hline CPS - 0,5\% & $03 / 07 / 2018$ & $04 / 07 / 2018$ \\
\hline CPS - 1,0\% & $04 / 07 / 2018$ & $05 / 07 / 2018$ \\
\hline TRAÇOS & $\begin{array}{c}\text { ROMPIMENTO } \\
\text { AOS 07 DIAS }\end{array}$ & $\begin{array}{c}\text { ROMPIMENTO } \\
\text { AOS } 28 \text { DIAS }\end{array}$ \\
\hline REFERÊNCIA & $10 / 07 / 2018$ & $31 / 07 / 2018$ \\
\hline CPS - 0,5\% & $11 / 07 / 2018$ & $01 / 08 / 2018$ \\
\hline CPS - 1,0\% & $12 / 07 / 2018$ & $02 / 08 / 2018$ \\
\hline
\end{tabular}

Fonte: Autores, (2018). 


\section{III.2 PORCENTAGEM DE ABSORÇÃO DE ÁGUA POR IMERSÃO DOS CORPOS DE PROVA AOS 28 DIAS}

O resultado da análise de imersão dos corpos de prova após o processo de cura úmida estará representado na Tabela 3 e Figura 9 de acordo com os resultados obtidos aos 28 dias de cura do concreto, o primeiro resultado é verificado logo após o desmolde dos corpos de prova com o auxílio de uma balança digital e o segundo é feito antes da ruptura, os dados são coletados e comparados de acordo com o traço proposto em porcentagem.

Tabela 3: Verificação da absorção de água e média de absorção do concreto aos 28 dias de cura úmida.

\section{Absorção de água do concreto aos 28 dias}

\begin{tabular}{|c|c|c|c|c|}
\hline \multirow{2}{*}{ Mistura } & $\begin{array}{c}\text { Massa } \\
\text { seca } \\
(\mathrm{g})\end{array}$ & $\begin{array}{c}\text { Massa } \\
\text { saturada } \\
(\mathrm{g})\end{array}$ & $\begin{array}{c}\text { Absorção } \\
(\%)\end{array}$ & $\begin{array}{c}\text { Média } \\
(\%)\end{array}$ \\
\hline \multirow{2}{*}{ REFERENCIA } & 3698,3 & 3738,6 & 1,09 & \multirow{2}{*}{1,07} \\
\cline { 2 - 4 } & 3718,6 & 3757,7 & 1,05 & \\
\hline \multirow{2}{*}{ CPS - 0,5\% } & 3652,2 & 3703,9 & 1,42 & \multirow{2}{*}{1,33} \\
\cline { 2 - 4 } & 3612,6 & 3657,5 & 1,24 & \\
\hline \multirow{2}{*}{ CPS - 1,0\% } & 3599,9 & 3651,7 & 1,44 & \multirow{2}{*}{1,41} \\
\cline { 2 - 4 } & 3588,9 & 3638,8 & 1,39 & \\
\hline
\end{tabular}

Fonte: Autores, (2018).

Na Figura 9 é possível observar com mais clareza a diferença na média de porcentagem absorvida entre os três diferentes traços usados nesta pesquisa a Mistura REFERENCIA como já era esperado absorveu menos água que as misturas com adição e $0,5 \%$ e $1,0 \%$ do material orgânico. Mesmo com a absorção superior as demais, o traço com $1,0 \%$ ainda sim se mantem aceitável.

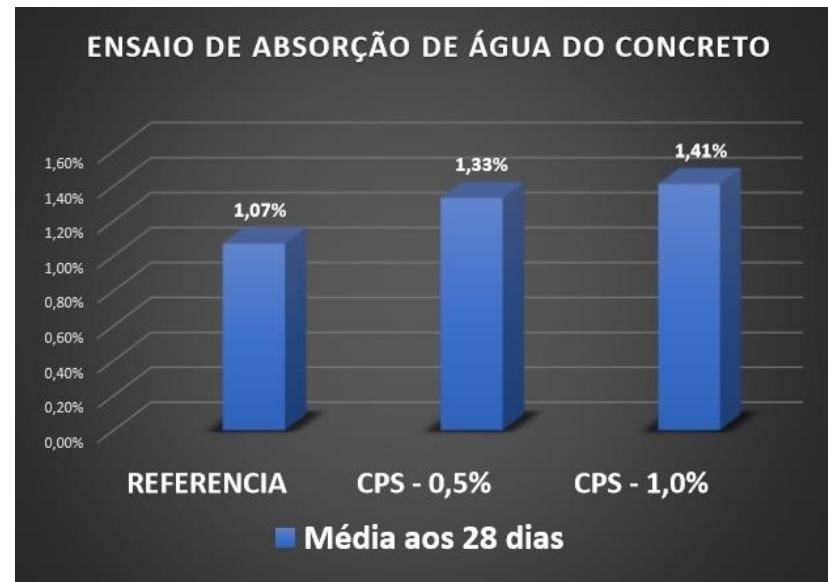

Figura 9: Gráfico da porcentagem média de absorção de água do concreto aos 28 dias

Fonte: Autores, (2018).

\section{III.3 RESISTENCIA À COMPRESSÃO AXIAL DOS CORPOS DE PROVA}

Os resultados das rupturas dos corpos de prova foram obtidos obedecendo os dias estipulados para esta pesquisa primeiramente em Kgf e depois transformados em MPa, a Tabela 4 mostra o resultado dos 12 corpos de prova rompidos nesta etapa, um par de amostras para cada dia estabelecido sendo assim dois para os 07 dias e dois para os 28 dias. O resultado é a média obtida em MPa retirada dessas amostras.

Tabela 4: Resistência média à compressão dos corpos e prova aos 07 e 28 dias do concreto endurecido

\begin{tabular}{|c|c|c|c|c|}
\hline \multirow{2}{*}{ Mistura } & \multicolumn{2}{|c|}{$\begin{array}{c}\text { Carga de ruptura } \\
(\mathrm{Kgf})\end{array}$} & \multicolumn{2}{|c|}{$\begin{array}{c}\text { Resistência à } \\
\text { compressão (Mpa) }\end{array}$} \\
\hline & 7 dias & 28 dias & $\begin{array}{c}7 \\
\text { dias }\end{array}$ & 28 dias \\
\hline \multirow{2}{*}{ REFERENCIA } & 19920,0 & 25110,0 & 25,37 & 31,98 \\
\hline & 21830,0 & 30460,0 & 27,80 & 38,79 \\
\hline \multirow{2}{*}{ CPS - $0,5 \%$} & 17480,0 & 20810,0 & 22,26 & 26,50 \\
\hline & 16280,0 & 27020,0 & 20,73 & 34,40 \\
\hline \multirow{2}{*}{ CPS - $1,0 \%$} & 12280,0 & 17430,0 & 15,64 & 22,20 \\
\hline & 11910,0 & 22030,0 & 15,17 & 28,05 \\
\hline Mistura & \multicolumn{2}{|c|}{$\begin{array}{c}\text { Médias aos } 07 \text { dias } \\
\text { (Mpa) }\end{array}$} & \multicolumn{2}{|c|}{$\begin{array}{c}\text { Médias aos } 28 \text { dias } \\
\text { (Mpa) }\end{array}$} \\
\hline REFERENCIA & \multicolumn{2}{|c|}{26,58} & \multicolumn{2}{|c|}{35,38} \\
\hline CPS - $0,5 \%$ & \multicolumn{2}{|c|}{21,50} & \multicolumn{2}{|c|}{30,45} \\
\hline CPS - $1,0 \%$ & \multicolumn{2}{|c|}{15,41} & \multicolumn{2}{|c|}{25,13} \\
\hline
\end{tabular}

Fonte: Autores, (2018).

No gráfico especificado na Figura 10 é possível observar a média obtida pela resistência mecânica dos corpos de prova aos 07 e 28 dias já em MPa a demonstração em blocos facilita a comparação das análises dispostas lado a lado classificando os três tipos de amostras e informando a carga obtida. É possível observar que aos 28 dias todos os traços estabelecidos alcançaram a resistência esperada e predeterminada de $25 \mathrm{MPa}$ conforme a dosagem do concreto calculada.

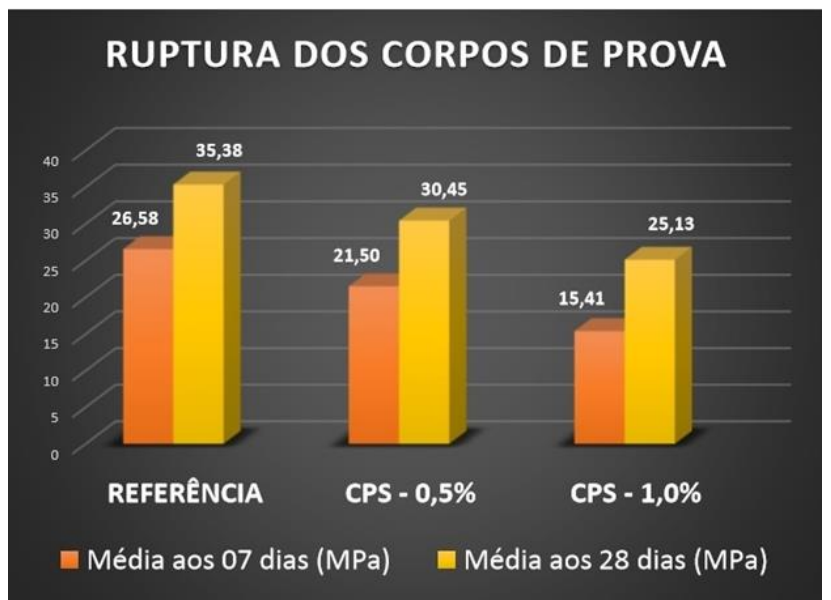

Figura 10: Gráfico de ruptura dos corpos de prova em MPa aos 07 e 28 dias de cura úmida do concreto endurecido.

Fonte: Autores, (2018). 


\section{CONCLUSÕES}

Com os resultados coletados ao longo de todo processo de estudo deste trabalho observamos que a resistência final dos corpos de prova dos traços CPS - $0,5 \%$ e CPS - $1,0 \%$ mesmo que abaixo do traço REFERÊNCIA ainda sim se mantem dentro do esperado de acordo com a norma. A partir dos dados fornecidos para compor o concreto e a escolha do traço levando em consideração o método da $\mathrm{ABCP}$ permitiu que o resultado fosse satisfatório. Podemos observar que o resultado dos testes a compressão mostrou-se similar aos valores encontrados em trabalhos com temas parecidos utilizando fibras naturais como agregados em substituição parcial e até mesmo como reforço em matrizes cimentícias. O trabalho contribuiu como uma forma de alternativa de agregado na substituição parcial do cimento utilizado na fabricação de concretos convencionais que futuramente a mesma poderá ser utilizada em obras de pequeno porte, para este caso com $25 \mathrm{MPa}$, a fibra natural teve um ótimo comportamento no resultado da ruptura dos corpos de prova. Portanto, para futuras pesquisas e para melhor verificação do resultado da utilização viável do material usado neste trabalho seria de grande importância verificar o comportamento do teste de carbonatação no traço usado para verificar o comportamento do concreto ao longo de sua vida e a chances que o mesmo teria de ocasionar fissuras.

\section{AGRADECIMENTOS}

Agradeço a minha família pelo incentivo prestado ao longo dessa trajetória universitária aos orientadores por todo o apoio técnico, aos professores do Centro Universitário do Norte (UNINORTE/LAUREATE) que ajudaram para a realização deste trabalho e à coordenação do curso de engenharia civil pela disponibilidade do laboratório de Materiais de Construção.

\section{REFERÊNCIAS}

[1] BUDINSKL, K,G. Engineering Materials: properties and selection. Prentice Hall International, 5ed. New Jersey, 1996.

[2] RIBEIRO, F. R.C.; RIBEIRO, J. M. S.; MOREIRA, K. M. V. Estudo de matrizes cimentícias reforçadas com fibras naturais e fibras sintéticas. Congresso Técnico Científico da Engenharia e da Agronomia (CONTECO), 2017. 74a SOEA - Semana Oficial de Engenharia e de Agronomia. Belém-PA.

[3] AGOPYAN, V; SAVASTANO JUNIOR, H. Fibras vegetais como materiais de construção. In: ISAIA, G. C. (editor) Materiais de construção civil e princípios de ciência e engenharia de materiais. São Paulo: Ibracon, 2007. 2v. 1721 p. AL ORAIMI S, SEIBI A. Mechanical characterization and impact behavior of concrete reinforced with natural fibres, Compos Struct, v. 32, p. 165-71, 1995.

[4] SILVA, E.; MARQUES, M.; FORNARI JUNIOR, C. Aplicação de fibra de coco em matriz cimentícias. 2012. Rev. Elet. em Gestão, Educação e Tecnologia Ambiental Universidade Federal de Santa Maria, UFSM/ RS. v.8. n ${ }^{\circ}$. p. 1555-1561. 2012.
[5] FARUK, O.; BLEDZKI, A. K.; FINK, H. P.; SAIN, M. Biocomposites reinforced with natural fibers: 2000-2010, Prog Polym Sci, 2012.

[6] IBGE - Instituto Brasileiro de Geografia e Estatística. Levantamento sistemático da produção agrícola. Rio de Janeiro; IBGE, v. 23, p. 1-80, 2010.

[7] SAVASTANO JUNIOR, H. Materiais à base de cimento reforçados com fibra vegetal: reciclagem de resíduos para a construção de baixo custo. Tese de livre-docencia, Escola Politécnica, Universidade de São Paulo. São Paulo, 2000.

[8] TORGAL, F. P,; JALALI, S. Cementitious building materials reinforced with vegetable fibres: Areview. Construction and Building Materials, www. elsevier.com/locate/conbuildmat, Agosto 2010.

[9] ABIKO,A.K;ORNSTEIN,S.W. Inserção Urbana e Avaliação Pós - Ocupação (APO) da Habitação de Interesse Social. Coletânea Habitare. São Paulo: FAUUSP, vol. 12002. 